\title{
MODELO PREDICTIVO DE CÁNCER DE PRÓSTATA EN PACIENTES CON INDICACIÓN DE BIOPSIA
}

\author{
R.A. MEDINA LÓPEZ*, R. BARRERO CANDAU*, A. MORALES LÓPEZ*, \\ E. SÁNCHEZ GÓMEZ*, A. CAYUELA**, J.L. PASCUAL DEL POBIL MORENO* \\ *Unidad de Uro-oncología. Servicio de Urología. ** Unidad de Investigación. \\ Hospital Universitario Virgen del Rocío. Sevilla.
}

Actas Urol Esp. 27 (5): 356-360, 2003

\section{RESUMEN}

"MODELO PREDICTIVO DE CÁNCER DE PRÓSTATA EN PACIENTES CON INDICACIÓN DE BIOPSIA"

OBJETIVO: Intentar conocer la probabilidad de padecer un carcinoma de próstata (CP) en base a la edad, tacto rectal (TR) y PSA, una vez indicada la realización de biopsia, con el fin de poseer mayor información a la hora de enfrentarnos, médico y paciente, a dicho problema.

MATERIAL Y MÉTODO: Estudio retrospectivo de 633 biopsias prostáticas, analizando edad, TR, nivel de PSA y resultado histológico. Los datos fueron incluidos en una base de datos de Access, analizándolos mediante el programa SPSS con el que se realizó un análisis de regresión logística.

RESULTADOS: Una vez indicada la biopsia, el TR es la variable con mayor poder de discriminación, con una odd ratio ajustada de 5,9 (IC 95\%, 3,9-8,9). El modelo matemático obtenido posee una sensibilidad del 57\% y una especificidad del $84 \%$. La probabilidad pretest fue del $36 \%$, pasando al $70 \%$ postest, con un valor predictivo negativo del $77 \%$ y positivo del $67 \%$.

CONCLUSIONES: El modelo matemático obtenido determina de forma individualizada la probabilidad de padecer un CP, y a su vez, más ajustada que la derivada de cumplir los criterios de biopsia. Una vez indicada la biopsia, la variable con mayor peso en la probabilidad de padecer CP es el TR, con independencia del PSA y la edad. El valor predictivo positivo del modelo es superior al del PSA o del TR utilizados de forma aislada.

PALABRAS CLAVE: Cáncer de próstata. Biopsia de próstata. Probabilidad. Modelo predictivo.

\section{ABSTRACT}

"PREDICTIVE MODEL FOR PROSTATE CANCER IN PATIENTS WITH BIOPSY INDICATION"

OBJETIVE: Attemp to determine the probability of developing prostate carcinoma taking ento acc age, digital rectal examination and PSA once a transrectal biopsy has been indicated, so that both doctors and patients have mor information to face such pathology.

MATERIAL AND METHODS: Retrospective study of 633 biopsies, takin ento acc the patient's age, digital rectal examination, PSA level and histology. The data were included in a database created with Access and were put a logistic regression by meens the softwere programe SPSS.

RESULTS: Once the biopsy is indicated, digital rectal examination is the parameter offesing a higher discriminatory valuer with an odd ratio of 5.9 (CI 95\%, 3.9-8.9). The mathematical model obtenned shows a sensitivity level of $57 \%$ and a level of specificity of $84 \%$. Pre-test probability is $36 \%$, the probability post-test incresang up to $70 \%$, and a negative predictive value of $77 \%$ and a positive predictive value of $67 \%$.

CONCLUSIONS: The mathematical model obtained individually determines the probability of suffeng from prostatic carcinoma. Moreover, using this model the probabilities obtained re more precise than those derived from the fact of fulfilling the criteria for a prostatic biopsy. Once a biopsy is indicated, the rectal examination becomes the parameter with a higher predictive value of PC, irrespective of PSA and age. The PPV of the model is higher than of the PSA or the digital recta examination used separately. 
$\mathrm{E}^{\prime}$ cáncer de próstata (CP) ocupa en los varones andaluces el tercer lugar en mortalidad por neoplasia, con una tasa bruta de 20,4/ 100.000 habitantes, precedido sólo por los tumores de pulmón y de colon. Su incidencia se sitúa en 2.400 nuevos casos al año, con una tasa bruta de 67/100.000 habitantes, ocupando igualmente el tercer lugar ${ }^{1}$. En la Unión Europea la incidencia se sitúa en 85.000 nuevos casos al año, representando aproximadamente el $9 \%$ de los fallecimientos por cáncer en los varones ${ }^{2}$.

Hasta hace unos años, el 60-65\% de los pacientes diagnosticados de CP padecían la enfermedad en estadio diseminado en el momento del diagnóstico, presentando metástasis óseas y/o linfáticas. En la actualidad, gracias a los métodos bioquímicos y técnicas de imagen, este porcentaje comienza a descender llamativamente, de ahí, la importancia de un diagnóstico precoz del CP.

En la práctica diaria los parámetros fundamentalmente empleados para la orientación diagnóstica del CP en un paciente con sintomatología miccional baja, son el tacto rectal (TR) y la determinación del antígeno prostático específico (PSA). Dependiendo de sus resultados se decidirá la indicación de una biopsia transrectal ecodirigida que confirmará el diagnóstico ${ }^{3}$.

La literatura urológica sigue mostrándonos amplias diferencias sobre el punto de corte a tomar en el nivel de PSA a la hora de indicar una biopsia prostática. Esto ha dado lugar a que en muchos protocolos se incluya el porcentaje de PSA-libre, el PSA ajustado a la edad, velocidad y densidad de PSA y el uso de nomogramas predictivos $^{4,5}$.

Por otro lado, la sociedad actual nos exige transmitir al paciente una completa información sobre su patología, con el fin de decidir, de forma compartida, las actitudes diagnóstico-terapéuticas a tomar.

$\mathrm{El}$ objetivo de este trabajo es intentar conocer la probabilidad que tiene un paciente de padecer un CP en base a su edad, TR y nivel de PSA, una vez indicada la realización de una biopsia transrectal, con el fin de poseer mayor información a la hora de enfrentarnos, médicos y pacientes, al problema.

\section{MATERIAL Y MÉTODO}

Se han estudiado de forma retrospectiva 633 biopsias transrectales prostáticas ecodirigidas realizadas en nuestro Servicio entre octubre de 2000 y febrero de 2002, analizando la edad del paciente, TR, nivel de PSA y resultado histológico.

Se indicó la realización de biopsia siempre que se cumpliera uno o más de los siguientes criterios:

- TR sospechoso de neoplasia.

- PSA sérico total mayor de $10 \mathrm{ng} / \mathrm{ml}$

- PSA libre $\leq$ al $18 \%$, si el PSA total se sitúa entre 4 y $10 \mathrm{ng} / \mathrm{ml}$.

Se excluyeron aquellos pacientes mayores de 80 años o que padecían alguna patología concomitante que contraindicara la realización de la prueba.

Se empleó un transductor multiplanar de 7 $\mathrm{MHz}$ y canal de punción integrado. Las muestras fueron tomadas mediante punción automática con aguja de $22 \mathrm{~mm}$.

Todos los pacientes siguieron la misma preparación, consistente en la administración de un enema de $250 \mathrm{cc}$. y la toma de $400 \mathrm{mg}$ de pefloxacino la noche antes y la misma mañana de la prueba.

A todos se les tomó 2 cilindros de cada lóbulo y uno de la zona central, así como una muestra de cualquier área ecográficamente sospechosa, lo que hacen un mínimo de cinco cilindros.

Los datos obtenidos fueron incluidos en una base de datos creada en el programa Access, analizándolos posteriormente mediante el paquete informático SPSS. Las variables cuantitativas, dado que no seguían una distribución normal, se expresaron mediante percentiles. Su comparación según histología y resultado de TR se efectuó mediante el test de la U de Mann Whitney. Para controlar el efecto de la edad y el valor del PSA fueron incluidas en el modelo de regresión logística junto al TR. El nivel de significación se fijó en el $5 \%$.

Se realizó un análisis de regresión logística.

\section{RESULTADOS}

Referente a la estadística descriptiva de la población estudiada, la edad media fue de 67 años, con una desviación estándar de 7,8. La dis- 
tribución por intervalos de edades aparece reflejada en la Figura 1. El valor de PSA total de situó por debajo de $4 \mathrm{ng} / \mathrm{ml}$ en 105 pacientes, entre 4,1 y $10 \mathrm{ng} / \mathrm{ml}$ en 233 individuos y por encima de 10,1 ng/ml en 305 (Fig. 2). El TR fue patológico (sospechoso de neoplasia) en 216 casos. El resultado histológico resultó ser adenocarcinoma de próstata en 214 pacientes, lo que supone un $38 \%$ de la población estudiada. En el $68,2 \%$ de los individuos que poseían un TR patológico se confirmó el diagnóstico histológico de $\mathrm{CP}$, frente al $23,9 \%$ en aquellos que presentaban un TR normal.

$\mathrm{El}$ análisis demostró que, una vez indicada la biopsia, el TR es la variable con mayor poder de discriminación, con un odd ratio ajustada de 5,9 (IC 95\%, 3,9-8,9). La odd ratio de la edad se situó en 1,04 (IC 95\%, 1,01-1,06) y la del PSA en

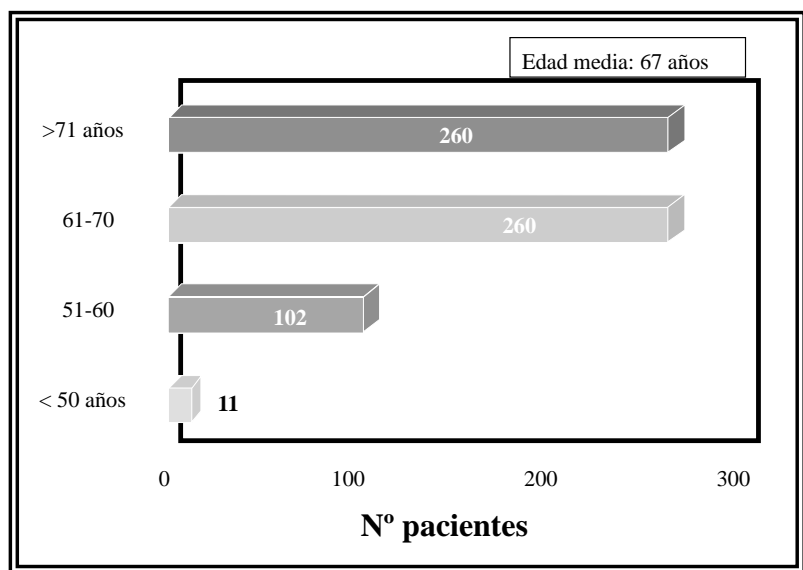

FIGURA 1. Distribución de los pacientes en intervalos de edad.

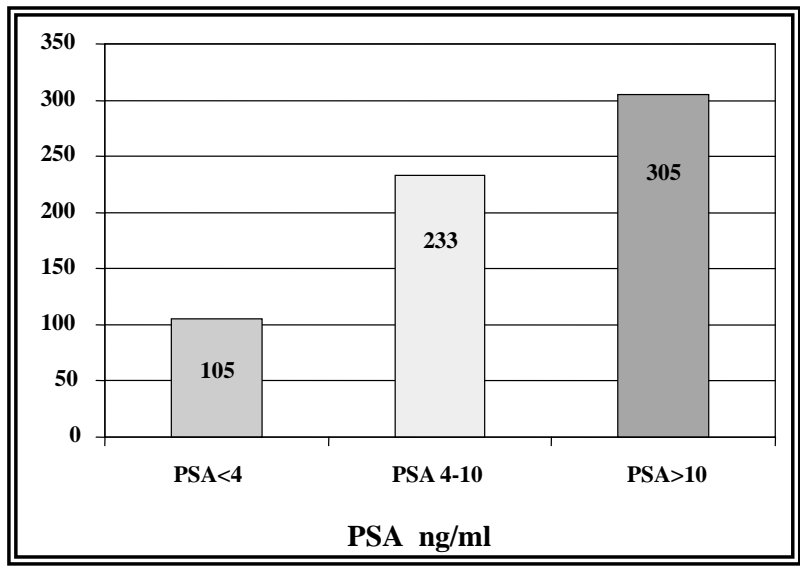

FIGURA 2. Distribución de la serie en intervalos de valor de PSA total.
1,002 (IC 95\%, 1,000-1,003). La distribución de la edad y PSA en relación al diagnóstico patológico y al TR se expresan en las Tablas I y II respectivamente, pudiendo observarse como los pacientes con adenocarcinoma prostático presentan una edad y un PSA total significativamente mayor que aquellos con patología no tumoral (Tabla I), al igual que ocurre en aquellos individuos con TR patológico frente a los que no lo poseen (Tabla II).

El modelo matemático de predicción obtenido mediante la regresión logística (Tabla III) es el expuesto a continuación, con una sensibilidad del $57 \%$ y una especificidad del $84,1 \%$.

\section{TABLA I}

DISTRIBUCIÓN DE LA EDAD Y EL PSA TOTAL SEGÚN EL RESULTADO HISTOLÓGICO DE LA BIOPSIA

\begin{tabular}{||l|l|c|c|c|c||}
\hline \multirow{2}{*}{ Edad } & & \multicolumn{4}{|c||}{ Percentiles } \\
\cline { 2 - 7 } & Histologia & 25 & 50 & 75 & \\
\cline { 2 - 7 } & No neoplasia & 61 & 67 & 72 & $\mathrm{p}<0,05$ \\
\cline { 2 - 7 } & Adenocarcinoma & 65 & 69 & 73 & \\
\hline \multirow{2}{*}{ PSA total } & No neoplasia & 6,01 & 8,96 & 15,45 & $\mathrm{p}<0,05$ \\
\cline { 2 - 7 } & Adenocarcinoma & 8,92 & 17,81 & 47,95 & \\
\hline
\end{tabular}

TABLA II

DISTRIBUCIÓN DE LA EDAD Y EL PSA TOTAL SEGÚN EL TACTO RECTAL

\begin{tabular}{||l|l|c|c|c|c||}
\hline \multirow{2}{*}{ Edad } & & \multicolumn{4}{|c|}{ Percentiles } \\
\cline { 2 - 6 } & Tacto rectal & 25 & 50 & 75 & \\
\cline { 2 - 7 } & Normal & 61 & 67 & 72 & $\mathrm{p}<0,05$ \\
\cline { 2 - 6 } & Patológico & 64,2 & 69 & 73 & \\
\hline \multirow{2}{*}{ PSA total } & Normal & 6,42 & 9,4 & 17,39 & $\mathrm{p}<0,05$ \\
\cline { 2 - 6 } & Patológico & 8,2 & 17,76 & 51,72 & \\
\hline
\end{tabular}

TABLA III

REGRESIÓN LOGÍSTICA.

(OR: odd ratio, IC: intervalo de confianza)

\begin{tabular}{|l|c|c|c|c||}
\hline \multirow{2}{*}{} & & \multicolumn{2}{|c|}{ I.C. 95\% para OR } & Significación \\
\cline { 3 - 4 } & OR & inferior & superior & estadística \\
\hline Edad & 1,040 & 1,012 & 1,069 & 0,005 \\
\hline PSA & 1,002 & 1,000 & 1,003 & 0,005 \\
\hline TR & 5,905 & 3,907 & 8,926 & 0,000 \\
Constate & 0,020 & & & 0,000 \\
\hline
\end{tabular}




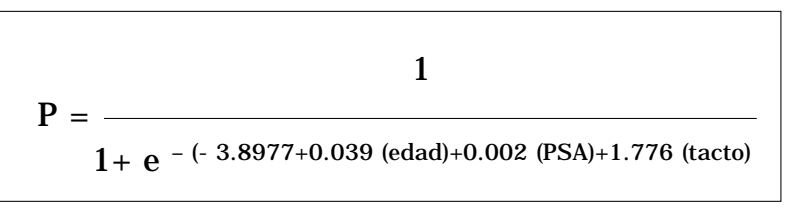

P: probabilidad de padecer $\mathrm{CP}(0 / 1)$

Edad: en años

PSA: valor de PSA total en $\mathrm{ng} / \mathrm{ml}$

Tacto: valor 0 si TR normal; valor 1 si TR patológico.

Aplicando esta fórmula a nuestros pacientes se calculó la probabilidad de padecer CP. Se consideró caso cuando esta probabilidad era $\geq 0,5, \mathrm{y}$ no caso cuando era inferior a este valor.

Según los datos analizados, y teniendo en cuenta los criterios de biopsia prostática empleados, la probabilidad pretest se sitúa en un $36 \%$, pasando al $70 \%$ de probabilidad postest, con un valor predictivo negativo (VPN) del $77 \%$ y positivo (VPP) del 67\%.

\section{DISCUSIÓN}

Existe un claro aumento de la incidencia de $\mathrm{CP}$, fundamentalmente por el envejecimiento de la población y la determinación del PSA en todo varón con sintomatología miccional baja. No obstante, sólo el 8\% de los casos se manifestarán clínicamente frente al resto que permanecerán latentes ${ }^{6}$.

Por otro lado, el concepto de decisión compartida entre paciente y médico está hoy extendiéndose progresivamente, con lo cual la demanda de información por parte de los enfermos es cada día mayor.

Se considera la determinación del PSA sérico el test con mayor VPP para el diagnóstico del CP, aunque el método más efectivo, que no el ideal, para su diagnóstico precoz, es su uso combinado con el TR ${ }^{7}$. El VPP se sitúa, según diferentes autores, entre el 31 y $34 \%^{8-10}$. Oesterling, en $1993^{11}$, introduce el concepto de PSA específico para la edad, basándose en la observación de que los niveles séricos del PSA ascienden con ésta, estimándose un aumento del 3,2\% anual de la concentración de PSA total.

De esta forma, debido a la baja especificidad del PSA, sobre todo en pacientes con TR normal y PSA $<10 \mathrm{ng} / \mathrm{ml}$, se le ha relacionado con otros parámetros para aumentar dicha especificidad ${ }^{12}$.
Todo ello, unido a la discusión sobre el punto de corte de PSA a considerar para la indicación de una biopsia, ha hecho introducir a grupos de trabajo como el del Johns Hopkins Hospital ${ }^{4}$, la utilización de nomogramas basados en el valor de PSA, el TR y la edad del paciente; obtienen así una información personal más ajustada, lo que facilita la información a transmitir y la pertinente toma de decisión. Este estudio se llevó a cabo mediante screening de la población, a diferencia del nuestro, realizado sólo sobre pacientes con indicación de biopsia prostática al cumplir los criterios ya expuestos, lo que probablemente explica la baja sensibilidad del modelo. De una u otra forma, tanto en el análisis realizado por este grupo como en el nuestro, la variable con mayor potencia para el riesgo de padecer un CP es el TR, aunque la odd ratio mostrada por nuestro análisis $(5,9)$ es superior a la publicada por ellos $(3,8$ (IC 95\%, 2,8-4,9)). Este dato fue igualmente corroborado por otro análisis de regresión logística publicado en 1999 por Virtanen ${ }^{13}$.

Con este modelo predictivo, al conjugar el valor del PSA, el TR y la edad del paciente, obtenemos un VPP del 67\%. Ello es importante si tenemos en cuenta que el VPP del PSA aislado, como se ha comentado, se sitúa en torno al $32 \%^{8-10}$ y el del TR oscila entre el 6 y el $39 \%^{14}$. Podremos conocer, con mayor precisión, la probabilidad que tiene un paciente de padecer un CP una vez que cumpla los criterios establecidos para la realización de una biopsia prostática, con una probabilidad postest del $70 \%$, frente a una probabilidad pretest (establecida por los criterios de biopsia) del $36 \%$. Es decir, cuando a un paciente le indicamos una biopsia, de forma global podemos informarle que presenta una probabilidad del $36 \%$ de padecer una neoplasia; sin embargo, al aplicar el modelo matemático obtendremos la probabilidad que posee dicho individuo con sus parámetros personales, de padecer un tumor, con una posibilidad de certeza del 70\%. La calidad de la información a transmitir mejora ostensiblemente con estos datos.

La fórmula obtenida mediante este análisis de regresión logística puede ser introducida en un programa informático con hoja de cálculo de forma que su utilización se simplifica hasta el nivel de una simple calculadora. 


\section{CONCLUSIONES}

El modelo matemático obtenido integra el valor de PSA, el tacto rectal y la edad del paciente, determinando de forma individualizada la probabilidad de padecer una neoplasia prostática, y a su vez, más ajustada que la derivada de cumplir los criterios para la indicación de biopsia prostática.

Una vez indicada la biopsia, la variable con mayor peso en la probabilidad de padecer CP es el tacto rectal, con independencia del PSA y de la edad.

El VPP del modelo es superior al del PSA o del TR utilizados de manera aislada.

\section{REFERENCIAS}

1. Plan Integral de Oncología de Andalucía 2002-2006. Consejería de Salud de la Junta de Andalucía. Sevilla, 2002.

2. BLACK RJ, BRAY F, FERLAY et al.: Cancer incidence and mortality in the European Union: cancer registry data and estimates of national incidence for 1990. Eur J Cancer 1997; 33: 1075-1107.

3. Proceso Asistencial Integrado Hipertrofia Benigna de Próstata-Cáncer de Próstata. Consejería de Salud de la Junta de Andalucía. Sevilla, 2001.

4. POTTER SR, HORNIGER W, TINZI M, BARTSCH G, PARTIN AW.: Age, prostate specific antigen and digital rectal examination as determinants of probability of having prostate cancer. Urology 2001; 57: 11001104.

5. GION M, MIONE R, BARIOLI P et al.: Percent free prostate-specific antigen in assessing the probability of prostate cancer under optimal analytical conditions. Clin Chem 1998; 44 (12): 2462-2470.

6. CABALLERO J, MARCHAL C, PADILLA M y cols.: Aumento de la tasa cruda de la incidencia del cáncer de próstata en nuestro medio. Actas Urol Esp 2001; 25 (9): 624-627.
7. ORTIZ R, ADRIAZOLA M, TEJADA E y cols.: Estudio estadístico de probabilidad del tacto rectal, PSA total, densidad del PSA en el cáncer de próstata. Arch Esp Urol 2002; 55 (1): 35-40.

8. LABRIE F, DUPONT A, SUBURU R et al.: Serum prostate specific antigen as pre-screening test for prostate cancer. J Urol 1992; 147: 846.

9. CATALONA WJ, RICHIE JP, AHMANN FR et al.: Comparison of digital rectal examination and serum prostate specific antigen in the early detection of prostate cancer: results of a multicenter clinical trial of 660 men. J Urol 1994; 151: 1.283.

10. BRAWER MK, CHETNER MP, BEATIE J et al.: Screening for prostate carcinoma with prostate specific antigen. $J$ Urol 1992; 147: 841.

11. OESTERLING JE, COONER WH, JACOBSEN SJ et al.: Influence of patient age on the serum PSA concentration. An important clinical observation. Urol Clin North Am 1993; 20 (4): 671-680.

12. HERRANZ F, VERDU F, DIEZ JM y cols.: Utilidad de cociente PSA libre/PSA total en el diagnóstico de cáncer de próstata en pacientes sintomáticos con niveles de PSA entre 2,5 y $20 \mathrm{ng} / \mathrm{l}$. Actas Urol Esp 2000; 24 (1): 24-30.

13. VIRTANEN A, GOMARI M, KRANSE $\mathrm{R}$ et al.: Estimation of prostate cancer probability by logistic regression: free and total prostate-specific antigen, digital recta examination, and hereditary are significant variables. Clin Chem 1999; 45 (7): 987 994.

14. GERBER GS, CHODAK GW.: Digital rectal examination in the early detection of prostate cancer. Cancer 1991; 7: 739-745.

Dr. R.A. Medina López

Av. Kansas City, 30 - piso 1103

41007 Sevilla

(Trabajo recibido el 16 julio de 2002) 